\title{
Portable Hand Held Laser-induced Breakdown Spectroscopy (LIBS) Instrumentation for in-field Elemental Analysis of Geological Samples
}

\section{Giorgio S. Senesi}

Istituto di Nanotecnologia (NANOTEC), PLasMI Lab, Via Amendola, Bari, Italy

\section{Introduction}

The elemental analysis of minerals and rocks is a very important routine work in geo-environmental sciences, as it provides unique information on their chemical composition that, in turn, allows to derive a variety of growth parameters that are of fundamental importance to the understanding of geological and weathering processes. Besides scientific applications, the elemental analysis of geomaterials is of economic importance for the evaluation of their industrial quality and for cultural heritage studies to assess the status of indoor and outdoor monuments, archeological sites and geological outcrops. In situ geochemical analysis has a high potential value not only for the terrestrial field geologists, but also for the next generation of planetary surface explorers as already shown by the three Mars rover missions of the past decade, i.e. Pathfinder, Mars Exploration Rovers (MER) and Mars Science Laboratory (MSL).

Recently, several manufacturers have recognized the importance of increasing the mobility of instrumentation by miniaturizing technologies for in situ applications in industry, mining and planetary exploration. Although handheld instrumentation, such as the wellestablished X-ray fluorescence (XRF) spectroscopy, is already used currently for various applications, it is not yet of common use in geological sciences [1]. Certainly laboratory instruments will stand the best to provide precise and detailed analyses and high quality data; however they present some unsolvable disadvantages, including high cost and requirement of extensive sample preparation and long analysis time. Differently, the use of handheld instruments would provide directly in the field rapid and finalized preliminary data on the composition of samples before any further, in-depth, specific, detailed laboratory analysis. By deploying handheld instruments, the field geologist will be able to streamline operations in the field, gain a real-time geochemical understanding of the site, and decide which samples to collect for further laboratory analyses, so saving time and money and minimizing further return trips to the field site.

One of the main requirement for an handheld instrument in geochemical applications is to achieve the widest range of elements that can be analyzed, especially when dealing with spatially related sample sets. The efficient identification of mineral and rock types relies on the instrumental capacity to measure all major forming elements and as many trace elements as possible, so allowing to understand their formation processes and subsequent changes that may have occurred. As testing of geological materials by field portable instruments is often performed in field harsh conditions, an easy portability, high robustness and fast data acquisition are required, so to incorporate easily the analytical program into the existing workflows related to activities such as drilling, mining and processing.

In the last decades, the Laser-Induced Breakdown Spectroscopy (LIBS) technique has been applied increasingly and successfully to mineral and rock analyses and their environmental, cultural and social implications [2-6]. In particular, in the last years my research has focused mainly on LIBS applications to the analysis of geological samples, including gemstones, meteorites, rocks and soils [7-9], agricultural samples such as sewage sludges, fertilizers and

\section{Publication History:}

Received: November 25, 2017

Accepted: December 14, 2017

Published: December 16, 2017

\section{Keywords:}

Daikai station, Collapse mechanism, Middle columns, Seismic motion, Numerical analysis

plants [10-12], and cultural heritage samples [13,14]. The aim of this commentary is to briefly focus on how a handheld LIBS instrumentation fits the requirements outlined above, and on the recent progress achieved by the scientific geological community in its use to analyze different geomaterials.

\section{The LIBS technique}

The common LIBS system generally used in many applications employs a $\mathrm{Q}$ switched Nd-YAG laser that emits pulses at a wavelength of $1064 \mathrm{~nm}$, with a temporal width ranging from 5 to $20 \mathrm{~ns}$ and a maximum energy of $100 \mathrm{~mJ} /$ pulse at a repetition rate of up to 20 $\mathrm{Hz}$.The pulse energy can be adjusted manually using appropriate optics that ensures the analysis performed at irradiation conditions yielding spectral data with a minimal interaction with the sample. Nd:YAG lasers can also provide wavelengths at 532, 355, and 266 $\mathrm{nm}$, which are, however, less powerful. In general, the choice of the optimal wavelength is dictated by the sample material and by the type of application [15].

The plasma radiation is generally collected through a lens positioned non-collinearly with respect to the axis of sample irradiation, and focused on a fiber optic that transfers the light emission to a spectrometer. This instrument enables recording spectra generally within a wide range, from 200 to $800 \mathrm{~nm}$, and provides a spectral resolution of approximately $0.1 \mathrm{~nm}$. The spectrometer is interconnected and controlled by the same software and electronics that enable proper temporal operations, i.e. same delay and gate parameters, which is of great importance in a LIBS measurement. The laser operations are controlled by the spectrometer software and for each analysis, pulses are triggered manually in single shots or in a series of a predefined number of shots. In portable handheld LIBS instrument all components are miniaturized and the laser head and optics are safely enclosed in a rigid case.

${ }^{\text {}}$ Corresponding Author: Proff. Giorgio S. Senesi, Istituto di Nanotecnologia (NANOTEC), PLasMI Lab, Via Amendola 122/D, 70126 Bari, Italy; E-mail: giorgio.senesi@nanotec.cnr.it.

Citation: Senesi GS (2017) Portable Hand Held Laser-induced Breakdown Spectroscopy (LIBS) Instrumentation for in-field Elemental Analysis of Geological Samples. Int J Earth Environ Sci 2: 146. doi: https://doi.org/10.15344/2456$351 \mathrm{X} / 2017 / 146$

Copyright: @ 2018 Senesi. This is an open-access article distributed under the terms of the Creative Commons Attribution License, which permits unrestricted use, distribution, and reproduction in any medium, provided the original author and source are credited. 
Custom-made softwares based on a spectral database of standard materials are generally used for sample identification. For example, when studying minerals, pure mineral samples are analyzed preliminarily and reference spectra are collected and stored in a reference database. Then, in the analysis of a real sample or object, the software provides mineral identification by direct comparison of the major lines of the reference spectra with those of the unknown sample. Further, the final elemental identification can be performed by using the NIST reference database [16] of atomic emission lines, and cross-comparing the two results.

\section{Geological Application of Portable Handheld LIBS Instrument}

Portable, handheld LIBS instruments have been shown to be able to measure most major and trace elements in a broad variety of minerals, whereas this cannot be achieved by the use of other field portable instruments. In particular, light elements such as Li, C, B, and Na can be measured by LIBS but not by XRF. Further, LIBS features improved detection limits for light elements such as $\mathrm{Mg}, \mathrm{Al}$ and $\mathrm{Si}$ [17-19].

Connors et al. [17] demonstrated that handheld LIBS instruments are well suited to analyze geochemical sample sets of various origin and nature, including igneous rocks and soils, by enabling analyses traditionally limited to the laboratory to be performed in the field. These authors presented calibration data for multiple elements of interest along with examples of elemental mapping in heterogeneous samples. These preliminary results indicate the significant potential of LIBS to provide, not only the qualitative analysis and microelement mapping of heterogeneous samples, but also the quantitative concentration of many key elements. This performance, combined with GPS and wireless connection features, is expected to enable even more exciting field applications, such as physical mineral mapping across large areas and instant data transmission back to a central database [17].

Very recently, Harmon et al. [18] applied a commercially available, portable, handheld LIBS instrument to the rapid identification and discrimination of carbonate, silicate and oxide minerals and native gold, obtaining a high degree of confidence. The distinct chemical features of the samples analyzed, i.e. the spectral differences in minor and trace constituents between similar samples, were identified by using the principal component analysis (PCA) approach. Further, the partial least squares discriminant analysis (PLSDA) was used to distinguish and classify with excellent discrimination all sample types studied. These authors [18] have also discussed how the concept of geochemical fingerprinting can be applied to carbonate, silicate and oxide minerals and gold under simulated field conditions. This, by spectral matching with a spectral library predetermined and assembled for the target of interest in order to obtain mineral identification, stratigraphic correlation and provenance determination to be used in geochemical exploration.

The design of a commercial handheld LIBS instrument and the first test measurements performed on a fragment of a sedimentary rock monument have been presented in a very recent work by our research group [19].The main elemental composition of two distinct layers, i.e. the superficial black crust and the underlying limestone rock, could be rapidly identified and quantified with a high degree of confidence. A full broadband LIBS emission spectrum was recorded in a few seconds from a single laser shot, which provided information on the elements present in the weathered layer and underneath stone. Further, the application of Calibration Free (CF)-LIBS approach allowed to obtain a suitable quantitative composition of the main elements present in the samples.

\section{Conclusions and Perspectives}

The on-going advances in laser, spectrometer and detector technologies have contributed significantly to the development and construction of compact and user-friendly portable handheld LIBS instruments capable of operating outside the research laboratory. This has opened new opportunities to geologists, archaeologists and conservators by lowering the instrumentation cost that might be prohibitive for small and medium scale laboratories. For example, LIBS can be used to rapidly identify samples that may need special treatment and select appropriate samples in the field so optimizing further analysis to be performed in conventional laboratory programs. In the cultural heritage field handheld LIBS appears to be particularly promising for performing in-situ measurement of outdoor monuments, archaeological sites and mural and cave paintings, classifying objects, identifying surface alterations and/ or decide previously any further interventions at the conservation laboratory.

Despite handheld LIBS technology is apparently very promising for use in geological field investigations; the technology still needs important technological advances, including the increase of detection limits and improvements in analytical precision. Further, the user interfaces and calibrated materials are not yet designed for specific use in field geology, which renders difficult the rapid interpretation of geochemical data in the field. Commercial manufacturers of LIBS instruments are, however, refining their products by developing new and emerging technologies with the aim of expanding the scientific user base. In particular, more work is required to define the best operational parameters to be applied to a wider range of lithologies, the best practice for measuring trace elements, and refine sampling protocols to be used with handheld LIBS instrumentation. To this regard, appropriate algorithms should be developed which allow data obtained by handheld LIBS to yield reliable geochemical fingerprints for a variety of rock types in real time. Although the work done until now has shown that handheld LIBS is a valuable tool for terrestrial field geology, further investigation of the strengths, weaknesses, operational improvements and best practices for deploying the instrument, can be expected to advance extensively terrestrial field geology studies and become a critical tool for future planetary field explorers.

Further work is presently underway in our laboratories to optimize the handheld LIBS technique in order to obtain reliable quantitative analysis data of a quality level similar to that of other analytical techniques, such as electron probe microanalysis, and extend this approach to new areas of application.

\section{Competing Interests}

The authors declare that they have no competing interests.

\section{References}

1. YoungKE, EvansCA, HodgesKV, BleacherJE, Graff TG, et al. (2016) A review of the handheld $\mathrm{X}$-ray fluorescence spectrometer as a tool for field geologic investigations on Earth and in planetary surface exploration. Applied Geochemistry 72: 77-87. 
Citation: Senesi GS (2017) Portable Hand Held Laser-induced Breakdown Spectroscopy (LIBS) Instrumentation for in-field Elemental Analysis of Geological Samples. Int J Earth Environ Sci 2: 146. doi: https://doi.org/10.15344/2456-351X/2017/146

Page 3 of 3

2. Harmon RS, De Lucia FC, Miziolek AW, McNesby KL, Walters RA, et al. (2005) Laser-induced breakdown spectroscopy (LIBS) - an emerging field-portable sensor technology for real-time, in-situ geochemical and environmental analysis. Geochemistry: Exploration, Environment, Analysis 5: $21-28$.

3. Harmon RS, Russo RE, Hark RR (2013) Applications of laser-induced breakdown spectroscopy for geochemical and environmental analysis: a comprehensive review. Spectrochimica Acta B 87: 11-26.

4. Senesi GS (2014) Laser-Induced Breakdown Spectroscopy (LIBS) applied to terrestrial and extraterrestrial analogue geomaterials with emphasis to minerals and rocks. Earth-Science Reviews 139: 231-267.

5. Spizzichino V, Fantoni R (2014) Laser Induced Breakdown Spectroscopy in archeometry: A review of its application and future perspectives. Spectrochimica Acta B 99: 201-209.

6. Anglos D, Detalle V (2014) Cultural Heritage Applications of LIBS. In Musazzi S, Perini U(Eds) Laser-Induced Breakdown Spectroscopy. Springer Series in Optical Sciences, Springer, Berlin, Heidelberg.

7. Senesi GS, Dell'Aglio M, Gaudiuso R, De Giacomo A, Zaccone C, et al (2009) Heavy metal concentrations in soils as determined by laser-induced breakdown spectroscopy (LIBS), with special emphasis on chromium. Environmental Research 109: 413-420.

8. Rossi M, Dell'Aglio M, De Giacomo A, Gaudiuso R, Senesi GS, et al (2014) Multi-methodological investigation of kunzite, hiddenite, alexandrite, elbaite and topaz, based on laser-induced breakdown spectroscopy and conventional analytical techniques for supporting mineralogical characterization. Physics and Chemistry of Minerals 41: 127-140.

9. Senesi GS, Tempesta G, Manzari P, Agrosì G (2016) An Innovative Approach to Meteorite Analysis by Laser-Induced Breakdown Spectroscopy (LIBS). Geostandards and Geoanalytical Research 40: 533-541.

10. Senesi GS, Dell'Aglio M, De Giacomo A, De Pascale O, Chami ZA, et al. (2014) Elemental composition analysis of plants and composts used for soil remediation by Laser-Induced Breakdown Spectroscopy. Clean - Soil, Air, Water 42: 791-798.

11. Menegatti CR, Nicolodelli G, Senesi GS, Da Silva OA, Filho HJI, et al. (2017) Semiquantitative analysis of mercury in landfill leachates using double-pulse laser-induced breakdown spectroscopy. Applied Optics 56: 3730-3735.

12. Senesi GS, Romano RA, Marangoni BS, Nicolodelli G, Villas-Boas PR, et al. (2017) Laser-Induced Breakdown Spectroscopy associated with multivariate analysis applied to discriminate fertilizers of different nature. Journal of Applied Spectroscopy 84: 923-928.

13. Senesi GS, Carrara I, Nicolodelli G, Milori DMBP, De Pascale O, et al. (2016) Laser cleaning and laser induced breakdown spectroscopy applied in removing and characterizing black crusts from limestones of Castello Svevo, Bari, Italy: a case study. Microchemical Journal 124: 296-305.

14. Senesi GS, Nicolodelli G, Milori DMBP, De Pascale O (2017) Depth profile investigations of surface modifications of limestone artifacts by laserinduced breakdown spectroscopy. Environmental Earth Sciences 76: 565.

15. Cremers DA, Radziemski LJ (2006) Handbook of Laser-Induced Breakdown Spectroscopy. (2nd edition), Chichester: John Wiley \& Sons, UK.

16. https://www.nist.gov/pml/atomic-spectra-database

17. Connors B, Somers A, Day D (2016) Application of handheld LaserInduced Breakdown Spectroscopy (LIBS) to geochemical analysis. Applied Spectroscopy 70: 810-815.

18. Harmon RS, Hark RR, Throckmorton CS, Rankey EC, Wise MA, et al. (2017) Geochemical fingerprinting by handheld Laser-Induced Breakdown Spectroscopy. Geostandards and Geoanalytical Research, in press 41: 563-584

19. Senesi GS, Manzini D, De Pascale O (2017) Application of a laser-induced breakdown spectroscopy handheld instrument to the diagnostic analysis of stone monuments. 